\title{
THERMOELASTIC ANALYSIS OF THICK-WALLED FUNCTIONALLY GRADED SPHERICAL PRESSURE VESSELS WITH TEMPERATURE-DEPENDENT MATERIAL PROPERTIES
}

\author{
DÁvid GÖNCZI \\ Institute of Applied Mechanics, University of Miskolc \\ H-3515 Miskolc-Egyetemváros \\ mechgoda@uni-miskolc.hu
}

[Received: July 19, 2017; Accepted: September 14, 2017]

\begin{abstract}
The main objective of this paper is the determination of the displacement field and the associated stresses in spherical pressure vessels made of functionally graded materials which are subjected to axisymmetric thermal and mechanical loadings. The material properties are arbitrary functions of the radial coordinate and the temperature field. A numerical solution of this steady-state thermoelastic problem is presented which is based on a multilayered approach. The developed method can be used as an analytical solution for layered composite spherical bodies. The equations of the steady-state heat conduction and field equations of thermoelasticity are used for the problem, furthermore the original problem is solved as the superposition of two subproblems with simpler loads. The results of the developed solution are checked by an analytical solution where the linear thermal expansion is a specific function of the radial coordinate and the temperature, furthermore the Young modulus depends on the radial coordinate. The numerical results for functionally graded spheres under the action of constant pressure and temperature load are compared to the results of finite element simulation.
\end{abstract}

Mathematical Subject Classification: 74B05

Keywords: FGM sphere, thermoelastic stresses, temperature-dependent, multilayered.

\section{INTRODUCTION}

In recent decades, laminated or layered composite and functionally graded materials (FGM) have been widely used in numerous engineering applications due to their excellent material behavior. The concept of FGM was first considered in Japan in 1984 during a hypersonic space plane project. The body of the spaceplane is exposed to a very high temperature environment (about $2000 \mathrm{~K}$ ), with a temperature gradient of approximately $1000 \mathrm{~K}$, between the inside and outside of the spaceplane. At that time there was no uniform material able to endure such conditions. In a functionally graded material the composition and structure gradually change, resulting in a corresponding change in the material properties. These advanced materials have improved thermal resistance and mechanical properties. The smooth transition between the 
material properties decreases the chance of cracking and debonding under thermal and mechanical loads.

In recent years this concept has become more popular; papers and textbooks deal with the determination of thermal stresses and displacements within simple structural components (such as cylindrical and spherical vessels, disks) caused by axisymmetric steady-state temperature field and mechanical loading.

Books by Boley and Weiner [1], Lekhnitskii [2] and Lomakin 3] give solutions to many linearly elastic problem for non-homogeneous bodies. The analytical solutions for the stresses and displacements in spheres and cylinders made of functionally graded materials (radially graded) are given by Lutz and Zimmerman [4]. Their paper considered thick spherical and cylindrical bodies under radial thermal loads where the composition of the constituent materials was linear. The work by Tutuncu and Ozturk [5] derived closed-form analytical solutions for the stresses in functionally graded cylindrical and spherical bodies, subjected to internal pressure alone. Radially varying inhomogeneous material properties were considered using material stiffness which obeys a simple power-law and stress distributions depending on an inhomogeneity constant.

There are a number of works where multilayered or heterogeneous structural components are analysed, for example in $[6] 9$. The paper of Obata and Noda 10$]$ studied a one-dimensional steady-state thermal stress problem for functionally graded hollow circular cylinders and hollow spheres by the use of a perturbation approach to achieve the effect of the composition on stresses and to design the optimum FGM hollow circular cylinder and hollow sphere, under different assumptions of temperature distributions. The unsteady-state thermal stress field of FG circular hollow cylinders based on a multilayered method and Green functions was presented by Kim and Noda [1].

Gönczi and Ecsedi [12] dealt with the steady-state thermoelastic problem of functionally graded disks where the material properties were arbitrary functions of the radial coordinate. Eslami et al. 13 investigated the thermal and mechanical stresses in hollow FGM spheres using a direct method to solve the heat conduction and Navier equations, while the temperature distribution was assumed to be a function of the radial coordinate.

Nayak et al. 14 developed an analytical solution to obtain the radial, tangential and effective stresses within thick spherical pressure vessels made of FGMs subjected to axisymmetric mechanical and thermal loads. The material properties of the vessel are assumed to be graded in the radial direction based on the power-law function of the radial coordinate but the Poisson ratio has constant value. A work by Bayat at al. 15 dealt with the previously presented problem too and investigates the effect of the power-law index on the stress distribution. However these papers neglect the temperature dependence of the functionally graded materials.

The FGM concept can be applied to various structural components such as to pressure vessels. This paper deals with the numerical analysis of FGM spherical pressure vessels. We consider a spherical pressure vessel made of functionally graded material which is subjected to axisymmetric thermal and mechanical loads on the 


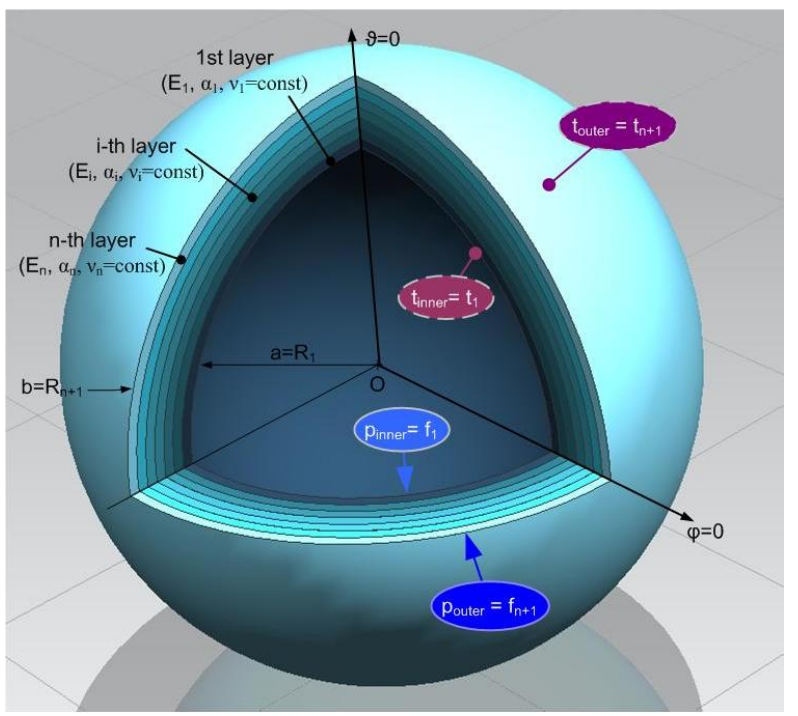

Figure 1. The multilayered model of the functionally graded sphere.

inner and outer boundary surfaces. This hollow functionally graded spherical body is subjected to unidirectional steady-state heat conduction with stress and thermal boundary conditions of the first kind on the boundary surfaces.

This paper presents a numerical method which approximates the thermoelastic problem of functionally graded spherical vessels with a problem of multilayered spherical bodies (Figure 1). The constant pressure is denoted by $p_{\text {outer }}$ which acts on the outer curved boundary surface while the uniformly distributed mechanical loading exerted on the inner surface is denoted by $p_{\text {inner. }}$. The radial stresses, the heatflow and the temperature field are all continuous functions of the radial coordinate $r$; furthermore the material properties are position- (radial coordinate) and temperaturedependent.

In our model the layers are made of homogeneous materials and are perfectly bonded, the material properties are constants within the layers but varying radially between them. The more layers are considered, the more accurate the computation will be (by FGMs: $n \rightarrow \infty$ ). Both the boundary conditions and the field equations [1, 16] are linear; therefore, the superposition principle can be used. This means that we can add the stresses and displacements caused by mechanical loads to the thermal stresses and displacements in order to solve this coupled problem. A spherical coordinate system is used to solve this problem.

\section{The FUnCtions of THE MATERIAL PROPERTIES}

Within the functionally graded material the volume fraction of the constituent materials gradually varies in the gradiation direction, thus the effective properties of FGMs change along this direction. Since functionally graded structures are most commonly 
used in high temperature environment where significant changes in mechanical properties of the constituent materials are to be expected [17,18, it is essential to take into consideration this temperature-dependency for accurate prediction of the mechanical response. Thus, the effective Young's modulus $E_{f}$, the Poisson ratio $\nu_{f}$, the linear thermal expansion coefficient $\alpha_{f}$ and the thermal conductivity $\lambda_{f}$ are assumed to be temperature dependent.

There are several methods to calculate these effective properties, such as the Mori-Tanaka scheme [19] for regions of the graded microstructure which have a welldefined continuous matrix and a discontinuous particulate phase or the self-consistent method [17, which assumes that each reinforcement inclusion is embedded in a continuum material and does not distinguish between matrix and reinforcement phases.

In many cases the effective material parameters can be expressed as nonlinear functions of the temperature field 17,20 .

$$
M(T)=P_{0}\left(P_{-1} T^{-1}+1+P_{1} T+P_{2} T^{2}+P_{3} T^{3}\right)
$$

In equation (1) $M(T)$ denotes the functions of the considered effective material properties $(E, \nu, \alpha$ and $\lambda), P_{0}, P_{-1}, P_{1}, P_{2}$ and $P_{3}$ are material dependent coefficients of the absolute temperature $(T[K])$. Using these results we can present functions for the temperature- and position-dependent functionally graded material properties of FGM spherical bodies and plates [17]:

$$
M_{f}(r, T)=\left[M_{1}(T)-M_{2}(T)\right][K]^{N}+M_{2}(T)
$$

where

$$
K^{\text {Sphere }}(r)=\frac{r-a}{b-a}, \quad \text { or } \quad K^{\text {Plate }}(z)=\frac{2 z-h}{2 h} .
$$

The indices 1 and 2 denote the constituent materials (mostly metal and ceramic components), $a$ and $b$ denote the inner and outer radii of the sphere, $h$ is the thickness of the plate, $z$ is the thickness coordinate and $N$ is the volume fraction of the FGM.

\section{Determination of the temperature Field}

At first we need to deal with the determination of the temperature field when the thermal conductivity is temperature- and radial coordinate-dependent $\lambda(T, r)$. We will approximate the temperature field of the functionally graded sphere for a temperature field of a multilayered spherical body with $n$ quasihomogeneous layers whose thermal conductivities depend only on temperature. It is recommended to partition the body according to the function of the material properties.

$$
R_{m i}=\frac{R_{i}+R_{i+1}}{2}, \quad \lambda_{i}(T)=\lambda\left(r=R_{m i}, T\right), \quad i=1 \ldots n .
$$

For this case the nonlinear differential equation for the temperature field of the $i$-th layer $T_{i}(r)$ has the following form:

$$
\frac{1}{r^{2}} \frac{d}{d r}\left[\lambda_{i}(T(r)) \cdot r^{2} \frac{d T_{i}(r)}{d r}\right]=0, \quad R_{i} \leq r \leq R_{i+1}, \quad i=1, \ldots, n .
$$


Using the Kirchhoff integral transformation, this problem becomes linear

$$
\theta=\int_{0}^{T_{i}} \lambda(\vartheta) d \vartheta, \quad \frac{1}{r^{2}} \frac{d}{d r}\left[r^{2} \frac{d \theta}{d r}\right]=0
$$

From the thermal boundary condition of first-kind the solution for the temperature field within the $i$-th layer can be derived in the following implicit form:

$$
\int_{t_{i}}^{T_{i}} \lambda_{i}(\vartheta) d \vartheta=\int_{t_{i}}^{t_{i+1}} \lambda_{i}(\vartheta) d \vartheta \frac{R_{i+1}}{R_{i}-R_{i-1}}\left(\frac{R_{i}}{r}-1\right), \quad i=1, \ldots, n
$$

We assume that the surface temperatures $t_{i}$ of the adjacent layers are equal and the radial heatflow $q$ is constant

$$
\begin{gathered}
t_{i+1}=T_{i}\left(R_{i+1}\right)=T_{i+1}\left(R_{i+1}\right), i=1, \ldots, n \\
{\left[\lambda_{i}\left(T_{i}(r)\right) \frac{d T_{i}(r)}{d r}\right]_{r=R_{i+1}}=q_{i}\left(R_{i+1}\right)=q_{i+1}\left(R_{i+1}\right)=} \\
=\left[\lambda_{i+1}\left(T_{i+1}(r)\right) \frac{d T_{i+1}(r)}{d r}\right]_{r=R_{i+1}}, i=1, \ldots, n-1 .
\end{gathered}
$$

After the manipulation of equations (5) the unknown $t_{i}(i=2, \ldots, n)$ boundary temperatures of the layers can be calculated from the following system of equations

$$
\text { const }=\int_{t_{i}}^{t_{i+1}} \lambda_{i}(\vartheta) d \vartheta \frac{R_{i+1} R_{i}}{R_{i}-R_{i-1}} \rightarrow t_{i} i=2, \ldots, n,
$$

moreover $t_{1}$ and $t_{n}+1$ temperatures are given. In the next step, instead of using equation (6) to compute the function of the temperature we will fit a curve or curves - for example with the least squares method - to the temperature values $t_{i}$ in order to make the further calculations easier especially the integrations. The approximation function has the following form:

$$
T_{a p p r}(r)=\theta_{-2} r^{-2}+\theta_{-1} r^{-1}+\theta_{0}+\theta_{1} r+\theta_{2} r^{2}
$$

In order to make the approximation more accurate more polynomial curves can be used to build the approximated temperature function. After the determination of the temperature field, the temperatures in the middle of the different layers are calculated for the approximation function of the material parameters.

$$
t_{m i}=T_{i}\left(r=R_{m i}\right), i=1, \ldots, n .
$$

\section{Approximation of the material parameters}

The second step is the approximation of the material properties $E(r, T(r)), \alpha(r, T(r))$ and $\nu(r, T(r))$ and the computation of their discrete values for the different homogeneous layers of the multilayered spherical model. We assign the following material 
properties for the spherical layers according to the functions of the material parameters $M$ :

$$
\begin{gathered}
L_{i v}=\left|\frac{M(a)-M(b)}{n}\right|, M(a)+(i-1) L_{i v}=M\left(R_{i}\right) \rightarrow R_{i}, 1=1 \ldots n+1 \\
E_{i}=E\left(r=R_{m i}, T=t_{m i}\right), \nu_{i}=\nu\left(r=R_{m i}, T=t_{m i}\right), \\
\alpha_{i}=\alpha\left(r=R_{m i}, T=t_{m i}\right), i=1 \ldots n .
\end{gathered}
$$

This means that we will approximate the arbitrary functions of the functionally graded material parameters with multistepped functions. To build the approximation function from the $n$ step we can use for example the Heaviside function. Figure 2 indicates a sketch of the $i$-th layer for the multilayered spherical body. The constant mechanical loads exerted on the inner and outer surfaces of the $i$-th layer are denoted by $f_{i}$ and $f_{i}+1$ respectively. The temperatures on the boundary surfaces are $t_{i}$ and $t_{i}+1$.

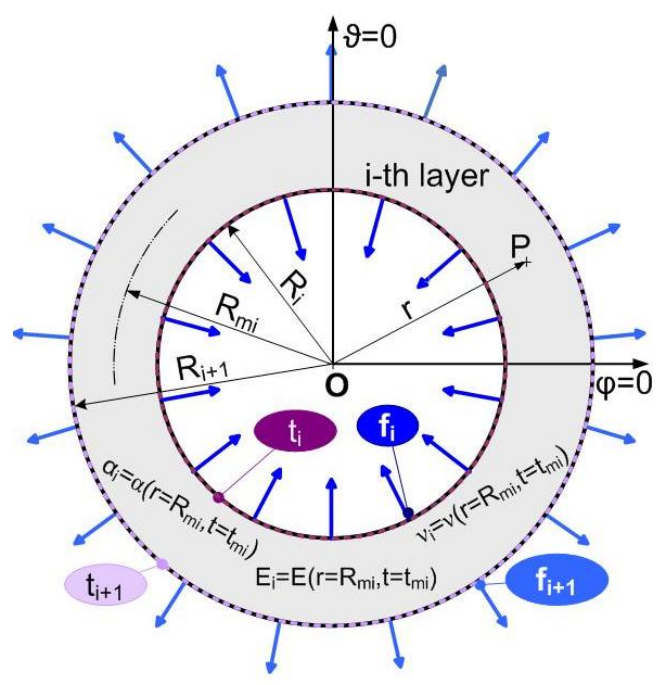

Figure 2. Sketch of the $i$-th layer with the mechanical and thermal loads

\section{The SOlUtion OF THE THERMOELASTIC PROBLEM}

In the next step the problem will be calculated in two parts, then the principle of superposition will be used to solve the problem. In the first case the $i$-th layer is under thermal loading $\left(t_{i}, t_{i}+1\right)$ and has the previously calculated steady-state temperature field, the stresses on the boundary surfaces $\left(f_{i}=f_{i}+1=0\right)$ of the layers have zero value. The thermal radial displacement $u_{i}^{T}(r)$ and the thermal stresses $\sigma_{i r}^{T}(r), \sigma_{i \varphi}^{T}(r)$, 
$\sigma_{i \theta}^{T}(r)$ have the following forms [1]:

$$
\begin{gathered}
u_{i}^{T}(r)=\frac{1+\nu_{i}}{1-\nu_{i}} \alpha_{i}\left[\frac{1}{r^{2}} \int_{R_{1}}^{r} r^{2} \tau_{i}(r) d r+\frac{2\left(1-2 \nu_{i}\right)}{1+\nu_{i}} \frac{r}{R_{i+1}^{3}-R_{i}^{3}} \int_{R_{1}}^{R_{2}} r^{2} \tau_{i}(r) d r+\right. \\
\left.+\frac{R_{i}^{3}}{R_{i+1}^{3}-R_{i}^{3}} \frac{1}{r^{2}} \int_{R_{1}}^{R_{2}} r^{2} \tau_{i}(r) d r\right] \\
\sigma_{i r}^{T}(r)=\frac{\alpha_{i} E_{i}}{1-\nu_{i}}\left[\frac{2}{r^{3}} \frac{r^{3}-R_{i}^{3}}{R_{i+1}^{3}-R_{i}^{3}} \int_{R_{1}}^{R_{2}} r^{2} \tau_{i}(r) d r-\frac{2}{r^{3}} \int_{R_{1}}^{r} r^{2} \tau_{i}(r) d r\right] \\
\sigma_{i \phi}^{T}(r)=\sigma_{i \vartheta}^{T}(r)=\frac{\alpha_{i} E_{i}}{1-\nu_{i}}\left[\frac{1}{r^{3}} \frac{2 r^{3}-R_{i}^{3}}{R_{i+1}^{3}-R_{i}^{3}} \int_{R_{1}}^{R_{2}} r^{2} \tau_{i}(r) d r-\frac{1}{r^{3}} \int_{R_{1}}^{r} r^{2} \tau_{i}(r) d r-\tau_{i}(r)\right] \\
i=1, \ldots, n,
\end{gathered}
$$

where $\tau_{i}(r)$ is the function of temperature difference of the $i$-th layer compared to a $t_{r}$ ef reference temperature. Because of the approximation of the temperature field, the integrals of equations 13 15 contain fourth degree polynomials $\left(r^{2} \tau_{i}(r)=\right.$ $\left.r^{2}\left(T_{a p p r}-t_{r e f}\right)\right)$ which can be easily calculated.

\section{Solution of the Elasticity problem}

In the second case it is assumed that the inner and outer boundary surfaces of the $i$-th spherical layer is under constant mechanical loading $\left(f_{i}\right.$ and $\left.f_{i}+1\right)$ without the thermal loads. The differential equation for the radial displacement field $u_{i}^{M}(r)$ can be derived from the equilibrium equations. The solution of this equation and the normal stresses have the following forms [1]:

$$
\begin{gathered}
u_{i}^{M}(r)=A_{i} r+\frac{B_{i}}{r^{2}} \\
\sigma_{i r}^{M}(r)=2 G_{i}\left(\frac{1+\nu_{i}}{1-2 \nu_{i}} A_{i}-\frac{2}{r^{3}} B_{i}\right), \\
\sigma_{i \vartheta}^{M}(r)=\sigma_{i \phi}^{M}(r)=2 G_{i}\left(\frac{1+\nu_{i}}{1-2 \nu_{i}} A_{i}+\frac{1}{r^{3}} B_{i}\right), i=1, \ldots, n .
\end{gathered}
$$

The unknown parameters $A_{i}$ and $B_{i}$ can be determined from the equations of the boundary conditions $\left(\sigma_{i r}^{M}\left(R_{i}\right)=f_{i}, \sigma_{i r}^{M}\left(R_{i+1}\right)=f_{i+1}\right)$ and we can use them to derive the expressions of the normal stresses.

$$
\begin{gathered}
A_{i}=\frac{\left(1-2 \nu_{i}\right)\left(R_{i+1}^{3} f_{i+1}-R_{i}^{3} f_{i}\right)}{2 G_{i}\left(1+\nu_{i}\right)\left(R_{i+1}^{3}-R_{i}^{3}\right)}, B_{i}=\frac{R_{i+1}^{3} R_{i}^{3}\left(f_{i+1}-f_{i}\right)}{4 G_{i}\left(R_{i+1}^{3}-R_{i}^{3}\right)}, \\
\sigma_{i r}^{M}(r)=\frac{R_{i+1}^{3} f_{i+1}-R_{i}^{3} f_{i}}{R_{i+1}^{3}-R_{i}^{3}}-\frac{R_{i+1}^{3} R_{i}^{3}\left(f_{i+1}-f_{i}\right)}{R_{i+1}^{3}-R_{i}^{3}} \frac{1}{r^{3}}, \\
\sigma_{i \phi}^{M}(r)=\frac{R_{i+1}^{3} f_{i+1}-R_{i}^{3} f_{i}}{R_{i+1}^{3}-R_{i}^{3}}-\frac{R_{i+1}^{3} R_{i}^{3}\left(f_{i}-f_{i+1}\right)}{2\left(R_{i+1}^{3}-R_{i}^{3}\right)} \frac{1}{r^{3}}, i=1, \ldots, n .
\end{gathered}
$$




\section{THE SUPERPOSITION OF THE THERMAL AND MECHANICAL LOADS}

The principle of superposition can be utilized for this problem, because both the previously used field equations and boundary conditions are linear. This means that we can add the stresses and displacements caused by mechanical loads 1620 to the thermal stresses and displacements 13 15 in order to solve this problem. For the computation of the radial displacement and radial and tangential stresses the following equations are used:

$$
\begin{gathered}
u_{i}(r)=u_{i}^{T}(r)+u_{i}^{M}(r), \sigma_{i r}(r)=\sigma_{i r}^{T}(r)+\sigma_{i r}^{M}(r), \\
\sigma_{i \phi}(r)=\sigma_{i \phi}^{T}(r)+\sigma_{i \phi}^{M}(r), i=1, \ldots, n .
\end{gathered}
$$

The unknown parameters $f_{i}(i=2, \ldots, n)$ in the equations of $u_{i}^{M}(r), \sigma_{i r}^{M}(r), \sigma_{i \varphi}^{M}(r)$ can be calculated from the following equations

$$
u_{i}\left(R_{i+1}\right)=u_{i+1}\left(R_{i+1}\right), i=1, \ldots, n-1,
$$

which ensure the continuity of the radial displacement field and furthermore $f_{1}$ and $f_{n+1}$ are given.

$$
\sigma_{1 r}\left(R_{1}\right)=f_{1}=-p_{1}, \sigma_{n r}\left(R_{n+1}\right)=f_{n+1}=-p_{n+1} .
$$

The system of equations 20 has the following form:

$$
a_{i} f_{i}+b_{i} f_{i+1}+c_{i} f_{i+2}=u_{i+1}^{T}\left(R_{i+1}\right)-u_{i}^{T}\left(R_{i+1}\right), i=2, \ldots, n-1 .
$$

where the constants $a_{i}, b_{i}$ and $c_{i}$ are

$$
\begin{gathered}
a_{i}=\frac{3 R_{i}^{3} R_{i+1}}{4 G_{i}\left(R_{i+1}^{3}-R_{i}^{3}\right)} \frac{\left(1-\nu_{i}\right)}{\left(1+\nu_{i}\right)}, \\
b_{i}=-\frac{R_{i+1}}{2 G_{i+1}\left(R_{i+2}^{3}-R_{i+2}^{3}\right)}\left[\frac{\left(1-2 \nu_{i+1}\right)}{\left(1+\nu_{i+1}\right)} R_{i+1}^{3}+\frac{R_{i+3}^{3}}{2}\right]- \\
-\frac{R_{i+1}}{2 G_{i}\left(R_{i+1}^{3}-R_{i}^{3}\right)}\left[\frac{\left(1-2 \nu_{i}\right)}{\left(1+\nu_{i}\right)} R_{i+1}^{3}+\frac{R_{i}^{3}}{2}\right], \\
c_{i}=\frac{3 R_{i+2}^{3} R_{i+1}}{4 G_{i+1}\left(R_{i+2}^{3}-R_{i+1}^{3}\right)} \frac{\left(1-\nu_{i+1}\right)}{\left(1+\nu_{i+1}\right)}, i=2, \ldots, n-1 .
\end{gathered}
$$

Using the previously determined parameters $f_{i}$ and equation (21) the radial displacement and the normal stresses of the multilayered spherical body can be evaluated. Due to the multilayered model the curve of the tangential normal stress may contain significant steps, but the stress values in the middle of each layer have good accuracy. Thus an approximate curve can be fitted to these points to increase the accuracy and convergence of the method. The recommended form of the approximate curve when $15>N>0.15$ :

$$
\sigma_{\phi-a p p r}(r)=F_{-3} r^{-3}+F_{-2} r^{-2}+F_{-1} r^{-1}+F_{0}+F_{1} r+F_{2} r^{2} .
$$




\section{An ANALYTiCAl SOLUtion}

An analytical solution is developed for the case when the Poisson ratio is constant, the distribution of the Young modulus is assumed to be described with a power-law along the radial coordinate [1], 15] the linear thermal expansion specifically depends on the temperature and radial coordinate, furthermore the temperature field has special form:

$$
\begin{gathered}
E(r)=P_{1}\left(\frac{r}{a}\right)^{m_{E}}, \alpha(r, T(r))=\left(P_{2}+P_{3} T(r)\right)\left(\frac{r}{a}\right)^{m_{\alpha}}, \\
T(r)=H_{1}-\frac{H_{2}}{r}, \text { if } \lambda=\text { const. }: \\
H_{1}=t_{\text {inner }}-\left(t_{\text {outer }}-t_{\text {inner }}\right) \frac{b}{a-b}, H_{2}=\left(t_{\text {outer }}-t_{\text {inner }}\right) \frac{a b}{a-b},
\end{gathered}
$$

where $P_{1}, P_{2}, P_{3}, m_{E}$ and $m_{\alpha}$ are material parameters. The strain-displacement and the stress-strain relations for spherical bodies can be expressed as [1, 16]:

$$
\begin{gathered}
\varepsilon_{r}=\frac{d u}{d r}, \quad \varepsilon_{\phi}=\frac{u}{r}, \\
\sigma_{r}(r)=\frac{E}{(1-2 \nu)(1+\nu)}\left[(1-\nu) \varepsilon_{r}+2 \nu \varepsilon_{\phi}-\alpha(1+\nu) T\right], \\
\sigma_{\vartheta}(r)=\sigma_{\phi}(r)=\frac{E}{(1-2 \nu)(1+\nu)}\left[\nu \varepsilon_{r}+\varepsilon_{\phi}-\alpha(1+\nu) T\right] .
\end{gathered}
$$

In this case the differential equation derived from the equilibrium equation for the radial displacement is

$$
\begin{gathered}
(1-\nu) \frac{d^{2} u}{d r^{2}}+\left(m_{E}+2\right) \frac{(1-\nu)}{r} \frac{d u}{d r}+2\left[\nu\left(m_{E}+1\right)-1\right] \frac{u}{r^{2}}= \\
=A_{1} r^{m_{\alpha}-1}+A_{2} r^{m_{\alpha}-2}+A_{1} r^{m_{\alpha}-3}
\end{gathered}
$$

where the constants $A_{1}, A_{2}$ and $A_{3}$ are

$$
\begin{gathered}
A_{1}=\frac{1+\nu}{a^{m_{\alpha}}}\left(-m_{E}-m_{\alpha}\right) H_{1}\left(P_{2}+P_{3} H_{1}\right), \\
A_{2}=\frac{1+\nu}{a^{m_{\alpha}}}\left(m_{E}+m_{\alpha}-1\right) H_{2}\left(P_{2}+2 P_{3} H_{1}\right), \\
A_{3}=\frac{1+\nu}{a^{m_{\alpha}}}\left(-m_{E}-m_{\alpha}+2\right) P_{3} H_{2}^{2} .
\end{gathered}
$$

The solution of the (34) differential equation:

$$
u(r)=C_{1} r^{\lambda_{1}}+C_{2} r^{\lambda_{2}}+\frac{G_{1} r^{m_{\alpha}+1}+G_{2} r^{m_{\alpha}}+G_{3} r^{m_{\alpha}-1}}{G_{4}},
$$

where $C_{1}$ and $C_{2}$ are unknown constants of integration and

$$
\begin{aligned}
& \lambda_{1}=\frac{1}{2}\left(-\left(m_{E}+1\right)+\sqrt{m_{E}^{2}+9+2 m_{E} \frac{1-6 \nu+5 \nu^{2}}{(\nu-1)^{2}}}\right), \\
& \lambda_{2}=\frac{1}{2}\left(-\left(m_{E}+1\right)-\sqrt{m_{E}^{2}+9+2 m_{E} \frac{1-6 \nu+5 \nu^{2}}{(\nu-1)^{2}}}\right),
\end{aligned}
$$




$$
\begin{gathered}
G_{1}=A_{1}\left\{2 \nu\left[\nu\left(m_{E}\left(3 m_{E}+5\right)+2\right)-m_{E}\left(m_{E}+6\right)-4\right]+\right. \\
+m_{\alpha}^{3}(\nu-1)^{2}\left(2 m_{E}+m_{\alpha}\right)+2\left(m_{E}+2\right)+m_{\alpha}(\nu-1)\left[m _ { \alpha } \left(m_{E}\left(1-m_{E}\right)+\right.\right. \\
\left.\left.+5+\nu\left(m_{E}\left(m_{E}-5\right)-5\right)-m_{E}\left(5 \nu\left(m_{E}+1\right)-5-m_{E}\right)\right]\right\} \\
G_{2}=A_{2}\left\{m_{\alpha}^{3}(\nu-1)^{2}\left(m_{\alpha}+2 m_{E}+2\right)+m_{E}\left[m_{E}(2 \nu-1)-2\right]+\right. \\
+m_{E} \nu^{2}\left(3 m_{E}+2\right)+m_{\alpha}(\nu-1)\left[-4 m_{E}\left(m_{E}+1\right)+m_{\alpha}\left(\nu \left(m _ { E } \left(m_{E}-\right.\right.\right.\right. \\
\left.\left.\left.-2)-5)-m_{E}\left(m_{E}+2\right)+5\right)\right]\right\}
\end{gathered}
$$

$$
\begin{aligned}
& G_{3}=A_{3}\left\{m_{\alpha}^{3}(\nu-1)^{2}\left(m_{\alpha}+2 m_{E}+4\right)+2 m_{E}\left[\nu^{2}\left(m_{E}+1\right)+m_{E} \nu-1\right]+\right. \\
& +m_{\alpha}(\nu-1) \cdot\left[m_{\alpha}\left(\nu\left(m_{E}+m_{E}^{2}+1\right)-m_{E}^{2}-5 m_{E}-1\right)-\right. \\
& \left.\left.-\left(\nu\left(m_{E}+1\right)\left(3 m_{E}+6\right)-m_{E}+m_{E}^{2}-6\right)\right]\right\}, \\
& G_{4}=\left[m_{\alpha}(\nu-1)\left(m_{\alpha}+m_{E}+3\right)-m_{E}(\nu+1)\right]\left[( \nu - 1 ) \left(m _ { \alpha } \left(m_{\alpha}+m_{E}-\right.\right.\right. \\
& \left.-1)-2)+m_{E}(1-3 \nu)\right]\left[(\nu-1)\left(m_{\alpha}\left(m_{\alpha}+m_{E}+1\right)-2\right)-2 m_{E} \nu\right] .
\end{aligned}
$$

Then the radial stress has the following form:

$$
\begin{gathered}
\sigma_{r}(r)=C_{1} S_{1} r^{\lambda_{1}+m_{E}-1}+C_{2} S_{2} r^{\lambda_{2}+m_{E}-1}+S_{0} r^{m_{\alpha}+m_{E}}+ \\
+S_{-1} r^{m_{\alpha}+m_{E}-1}+S_{-2} r^{m_{\alpha}+m_{E}-2}
\end{gathered}
$$

where

$$
\begin{gathered}
S_{1}=Z\left[(1-\nu) \lambda_{1}+2 \nu\right], S_{2}=Z\left[(1-\nu) \lambda_{2}+2 \nu\right], Z=\frac{P_{1}}{a^{m_{E}}(1-2 \nu)(1+\nu)}, \\
S_{0}=Z\left\{\frac{G_{1}}{G_{4}}\left[(1-\nu)\left(m_{\alpha}+1\right)+2 \nu\right]-\frac{H_{1}(1+\nu)\left(P_{2}+P_{3} H_{1}\right)}{a^{m_{\alpha}}}\right\}, \\
S_{-1}=Z\left\{\frac{G_{2}}{G_{4}}\left[(1-\nu) m_{\alpha}+2 \nu\right]+\frac{H_{2}(1+\nu)\left(P_{2}+2 P_{3} H_{1}\right)}{a^{m_{\alpha}}}\right\}, \\
S_{-2}=Z\left\{\frac{G_{3}}{G_{4}}\left[(1-\nu)\left(m_{\alpha}-1\right)+2 \nu\right]-\frac{H_{2}^{2}(1+\nu) P_{3}}{a^{m_{\alpha}}}\right\},
\end{gathered}
$$

The unknown constants $C_{1}, C_{2}$ can be obtained from the stress boundary conditions:

$$
\begin{gathered}
\sigma_{r}(a)=-p_{\text {inner }}, \quad \sigma_{r}(b)=-p_{\text {outer }}, \\
C_{1}=\frac{b^{\lambda_{2}+m_{E}-1} c_{a}-a^{\lambda_{2}+m_{E}-1} c_{b}}{S_{1}\left(a^{\lambda_{2}+m_{E}-1} b^{\lambda_{1}+m_{E}-1}-a^{\lambda_{1}+m_{E}-1} b^{\lambda_{2}+m_{E}-1}\right)}, \\
C_{2}=\frac{a^{\lambda_{1}+m_{E}-1} c_{b}-b^{\lambda_{1}+m_{E}-1} c_{a}}{S_{2}\left(a^{\lambda_{2}+m_{E}-1} b^{\lambda_{1}+m_{E}-1}-a^{\lambda_{1}+m_{E}-1} b^{\lambda_{2}+m_{E}-1}\right)}, \\
c_{a}=a^{m_{\alpha}+m_{E}}\left(S_{0}+S_{-1} a^{-1}+S_{-2} a^{-2}\right)+p_{\text {inner }}, \\
c_{b}=b^{m_{\alpha}+m_{E}}\left(S_{0}+S_{-1} b^{-1}+S_{-2} b^{-2}\right)+p_{\text {outer }} .
\end{gathered}
$$




\section{Analytical SOlUtion FOR The temperature FiELD}

An analytical solution is derived to check the accuracy of the method that calculates the temperature field (Section 2). The thermal conductivity is temperature- and coordinate-dependent and has the following special form:

$$
\lambda(r, T(r))=P_{1} e^{P_{2} T(r)}\left(\frac{r}{a}\right)^{m_{\lambda}},
$$

where $P_{1}, P_{2}$ and $m_{\lambda}$ are material constants. After solving equation (4) the temperature field can be calculated and its constants can be evaluated from the first-kind thermal boundary conditions:

$$
\begin{gathered}
T(r)=\frac{1}{P_{2}} \ln \left(\frac{P_{2} C_{1}}{\left(m_{\lambda}+1\right)} \frac{r^{-m_{\lambda}-1}}{a^{-m_{\lambda}}}-P_{2} C_{1} C_{2}\right) \\
C_{1}=\frac{a b(m+1)\left(e^{t_{\text {outer }} P_{2}}-e^{t_{\text {inner }} P_{2}}\right)}{P_{2}\left(a\left(\frac{b}{a}\right)^{-m_{\lambda}}-b\right)}, C_{2}=\frac{b e^{t_{\text {outer }} P_{2}}-a e^{t_{\text {inner }} P_{2}}\left(\frac{b}{a}\right)^{-m_{\lambda}}}{a(m+1)\left(e^{t_{\text {outer }} P_{2}}-e^{t_{\text {inner }} P_{2}}\right) b} .
\end{gathered}
$$

\section{NUMERICAL EXAMPLES}

This part of the paper deals with the verification of the developed methods. We used the equations of the previously presented analytical solutions and finite element simulation to verify the developed solutions. Furthermore, Maple 15 mathematical software was used to create the program of the developed method. In the first numerical example the accuracy of the calculation for the temperature field is investigated. The following data were used for equation 22 to carry out the numerical computation:

$$
\begin{gathered}
a=0.04 \mathrm{~m}, b=0.06 \mathrm{~m}, P_{1}=10 \frac{\mathrm{W}}{\mathrm{mK}}, P_{2}=1.34 \cdot 10^{-3} \frac{1}{\mathrm{~K}}, m_{\lambda}=1.9, \\
t_{\text {ref }}=273 \mathrm{~K}, t_{\text {inner }}=303 \mathrm{~K}, t_{\text {outer }}=623 \mathrm{~K}
\end{gathered}
$$

and the approximation function of the temperature field is built from two curves:

$$
\begin{gathered}
T_{\text {appr }}(r)=\left[\text { Heaviside }(r-a)-\text { Heaviside }\left(r-\frac{a+b}{2}\right)\right] T_{a p p r, 1} \\
+\left[\text { Heaviside }\left(r-\frac{a+b}{2}\right)-\text { Heaviside }(r-b)\right] T_{a p p r, 2}
\end{gathered}
$$

where $T_{a p p r, 1}$ and $T_{a p p r, 2}$ have the forms according to equation (10); furthermore, three cases with three different layer numbers $\left(n_{1}=5, n_{2}=9, n_{3}=17\right)$ are compared to the analytical solution of Eq. (54-55). Figure 3 shows the temperature function and the relative errors of the approximations when

$$
e_{M}(\%)=\left|\frac{M_{\text {analytical }}-M_{\text {numerical }}}{M_{\text {analytical }}}\right| \cdot 100, \quad M(r)=T(r), u(r), \sigma_{r}(r), \sigma_{\phi}(r) .
$$




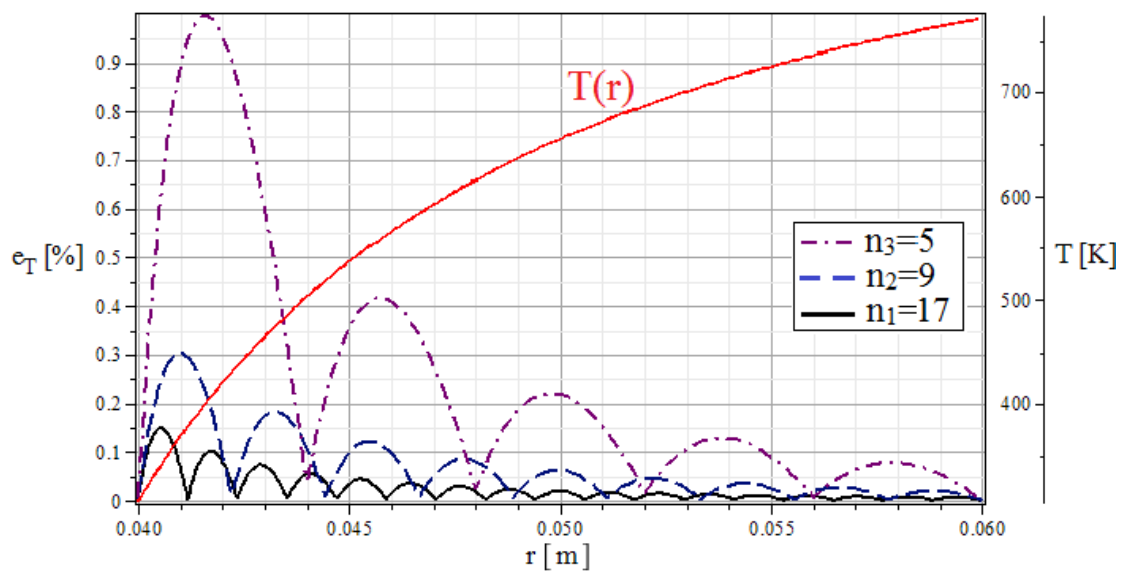

Figure 3. The temperature field and the relative errors of the model

In the second example the developed numerical method is compared to the analytical method of Section 8. The following numerical data were used to check the accuracy of the presented method:

$$
\begin{gathered}
a=0.065 m, b=0.08 m, P_{1}=200 G P a, P_{2}=10^{-8} \frac{1}{K^{2}}, P_{3}=2 \cdot 10^{-8} \frac{1}{K}, m_{\alpha}=1.9, \\
m_{E}=2, \lambda=58 \frac{W}{m K}, t_{\text {inner }}=723 K, t_{\text {outer }}=298 K, t_{\text {ref }}=273 K, \\
p_{\text {inner }}=20 M P a, p_{\text {outer }}=0 M P a .
\end{gathered}
$$
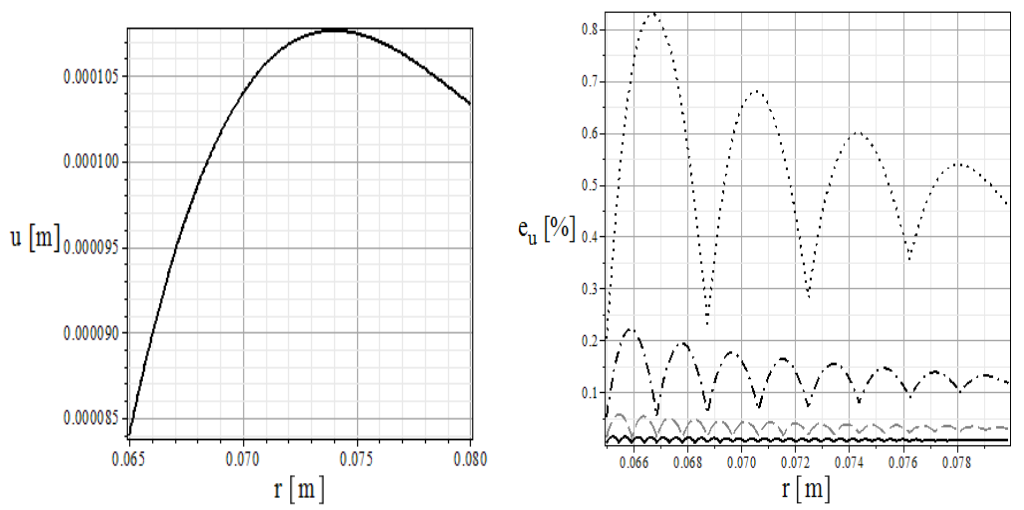

Figure 4. The radial displacement field and the relative errors $\left(n_{1}=\right.$ 4: dots, $n_{2}=8$ : dash-dot line, $n_{3}=16$ : dashed line, $n_{4}=32$ : solid line)

Figures (4 6) illustrate the radial displacement field, radial and tangential normal stresses, the convergence of the developed method and the relative errors $e_{u}(r), e_{\sigma r}(r)$, $e_{\sigma \varphi}(r)$ of the numerical model compared to the analytical solution. Four different layer 
numbers were used to build up the multilayered models: $n_{1}=4, n_{2}=8, n_{3}=16$ and $n_{4}=32$. Figure 6 shows the advantage of the approximation - solid continuous line - for the tangential stresses over the summarized - discontinuous line - curve.
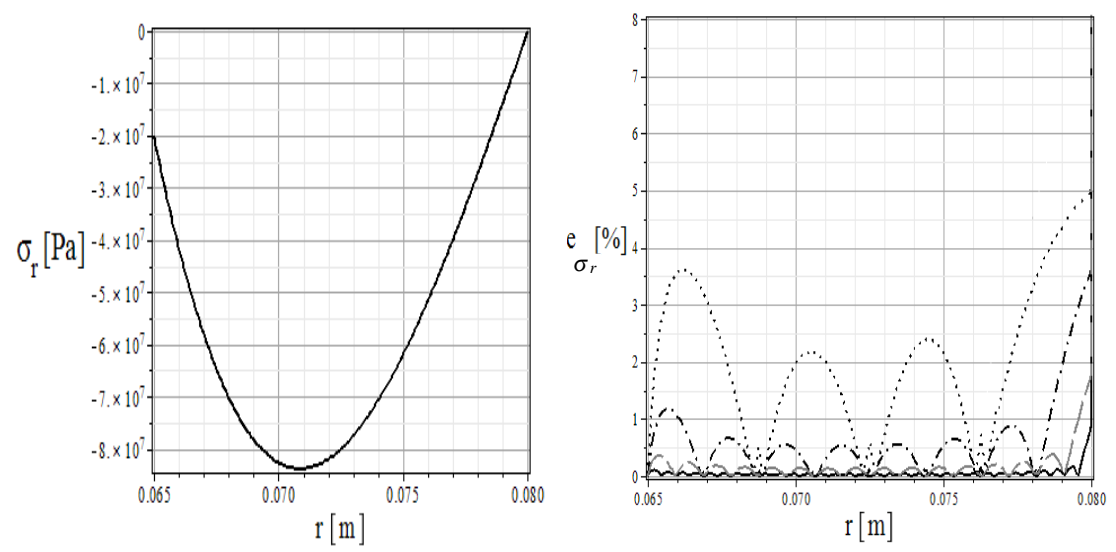

Figure 5. The radial normal stress and the relative errors $\left(n_{1}=4\right.$ : dots, $n_{2}=8$ : dash-dot line, $n_{3}=16$ : dashed line, $n_{4}=32$ : solid line)
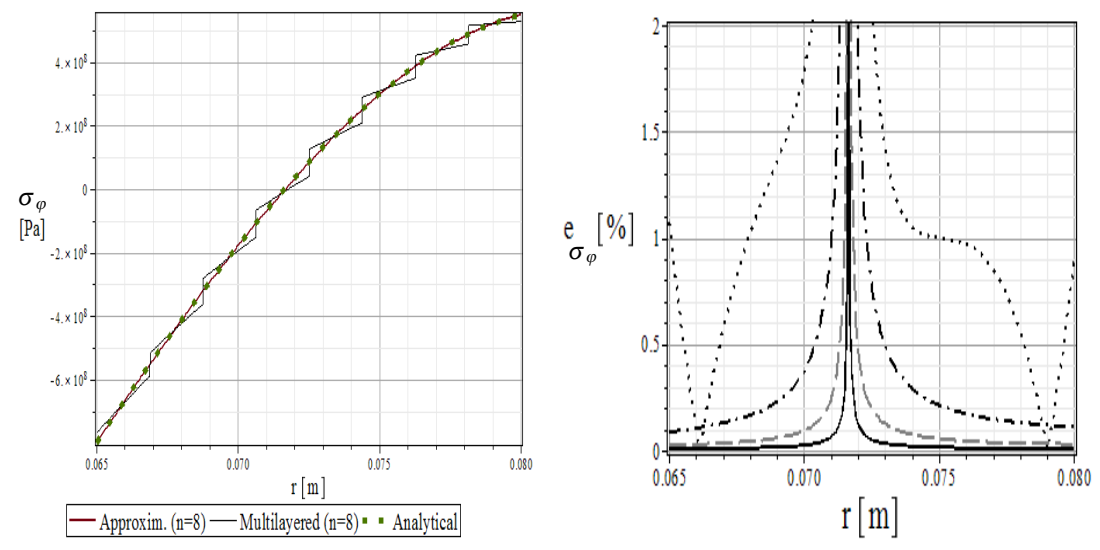

Figure 6 . The tangential stress and the relative errors $\left(n_{1}=4\right.$ : dots, $n_{2}=8:$ dash-dot line, $n_{3}=16:$ dashed line, $n_{4}=32:$ solid line)

In the next example a functionally graded spherical vessel is considered which is made from a steel-silicon nitride FGM and the effective material properties can be calculated by equations $(1,2)$. In this case the results of the multilayered method are verified by finite element simulation. The finite element model was created using a commercial FE code, ABAQUS CAE. In the FEM model - due to symmetry - only 
a quarter of the spherical body is created. The variation of the material properties was implemented by considering 30 layers with temperature-dependent material properties $\left(M_{i}=M\left(r=r_{i}, T(r)\right), i=1, \ldots, 30\right)$. An 8-node temperature-displacement, quadrilateral element was used to mesh the vessel, the number of elements is 4201 .

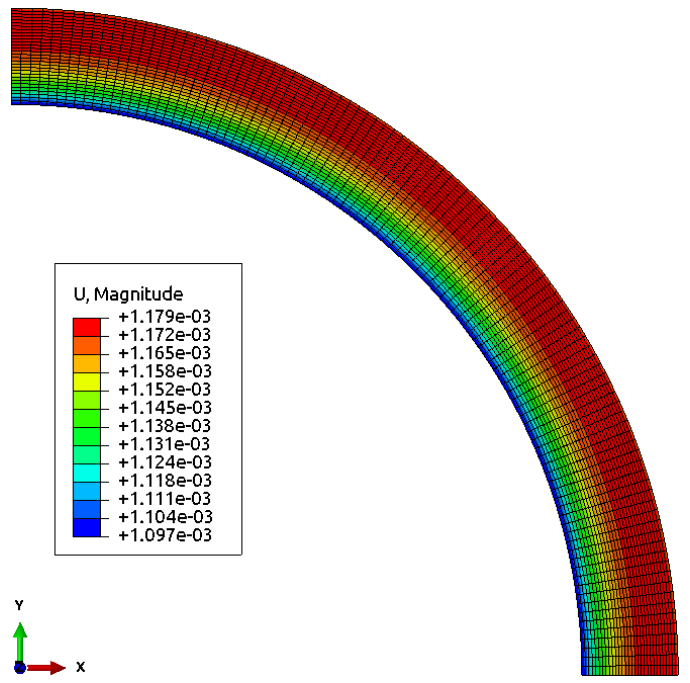

Figure 7. The finite element model and the displacement field

Figure 7 shows the mesh of the model on the deformed geometry with the displacement field. The material parameters [17], [18 can be found in Table 1, the geometry and the loads are:

$$
\begin{gathered}
a=0.5 \mathrm{~m}, b=0.59 \mathrm{~m}, t_{\text {inner }}=733 \mathrm{~K}, t_{\text {outer }}=303 \mathrm{~K}, t_{\text {ref }}=273 \mathrm{~K}, \\
p_{\text {inner }}=90 \mathrm{MPa}, p_{\text {outer }}=0 \mathrm{MPa} .
\end{gathered}
$$

Table 1. Material properties for the numerical example

\begin{tabular}{|c|c|c|c|c|c|c|c|c|}
\hline $\begin{array}{c}\text { material } \\
\text { property } \\
(P)\end{array}$ & \multicolumn{3}{|c|}{ metal (stainless steel) } & \multicolumn{3}{c|}{ ceramic (silicon nitrade) } \\
\cline { 2 - 9 } & $\mathbf{P}_{m 0}$ & $\mathbf{P}_{m 1}\left(10^{-3}\right)$ & $\mathbf{P}_{m 2}\left(10^{-3}\right)$ & $\mathbf{P}_{m 3}\left(10^{-3}\right)$ & $\mathbf{P}_{c 0}$ & $\mathbf{P}_{c 1}\left(10^{-3}\right)$ & $\mathbf{P}_{c 2}\left(10^{-3}\right)$ & $\mathbf{P}_{c 3}\left(10^{-3}\right)$ \\
\hline \hline$\lambda(\mathrm{W} / \mathrm{mK})$ & 15.39 & -1.264 & 20.92 & -7.223 & 12.723 & -1.032 & 5.466 & -7.876 \\
\hline$\alpha(1 / \mathrm{K})$ & $12.33 \cdot 10^{6}$ & 0.8086 & 0 & 0 & $3.873 \cdot 10^{6}$ & 0.9095 & 0 & 0 \\
\hline$E(\mathrm{~Pa})$ & $2.01 \cdot 10^{11}$ & 0.3079 & -6.5340 & 0 & $3.484 \cdot 10^{11}$ & -0.307 & 2.16 & -8.946 \\
\hline$\nu !$ & 0.3262 & -0.1 & 3.797 & 0 & 0.24 & 0 & 0 & 0 \\
\hline
\end{tabular}

Table 2 contains the results of the finite element simulation and the multilayered method when the volume fraction is $N=3$. In these case we note that if the temperature dependency of the material parameters is neglected, the relative errors are significant (for example if $M=M(r, T=298 K)=M(r)$, then the maximum error is $200 \mathrm{MPa}$ ). 
Table 2. Results of the developed method (ML) and the FE simulations

\begin{tabular}{|c|c|c|c|c|c|c|c|c|c|}
\hline$N=3$ & \multirow{2}{*}{$\begin{array}{l}\text { met- } \\
\text { hod }\end{array}$} & \multicolumn{2}{|c|}{$T(r)[\mathrm{K}]$} & \multicolumn{2}{|c|}{$u(r)[\mathrm{mm}]$} & \multicolumn{2}{|c|}{$-\sigma_{r}[\mathrm{MPa}]$} & \multicolumn{2}{|c|}{$-\sigma_{\varphi}[\mathrm{MPa}]$} \\
\hline$r(\mathrm{~mm})$ & & $n=30$ & $n=180$ & $n=30$ & $n=180$ & $n=30$ & $n=180$ & $n=30$ & $n=180$ \\
\hline \multirow{2}{*}{500} & $\mathrm{ML}$ & 733 & 733 & 1.09865 & 1.09869 & 90 & 90 & -330.17 & 330.23 \\
\hline & $\mathrm{FE}$ & 733 & & 1.09741 & & 90.1645 & & -332.17 & \\
\hline \multirow{2}{*}{518.04} & ML & 611.750 & 611.751 & 1.14211 & 1.14215 & 93.5435 & 93.5468 & 22.0908 & 22.142 \\
\hline & $\mathrm{FE}$ & 612.041 & & 1.14084 & & 98.8303 & & 19.650 & \\
\hline \multirow{2}{*}{536.07} & ML & 510.297 & 510.297 & 1.16493 & 1.16497 & 78.1207 & 78.1263 & 233.905 & 233.923 \\
\hline & $\mathrm{FE}$ & 510.604 & & 1.16352 & & 78.3125 & & 222.61 & \\
\hline \multirow{2}{*}{554.08} & ML & 426.717 & 426.718 & 1.17632 & 1.17636 & 54.3166 & 54.3210 & 341.202 & 341.216 \\
\hline & $\mathrm{FE}$ & 420.026 & & 1.17497 & & 54.5826 & & 325.583 & \\
\hline \multirow{2}{*}{572.08} & ML & 358.392 & 358.393 & 1.17974 & 1.17977 & 27.7325 & 27.7378 & 405.913 & 405.937 \\
\hline & $\mathrm{FE}$ & 358.703 & & 1.17846 & & 28.0633 & & 389.203 & \\
\hline \multirow{2}{*}{590} & ML & 303 & 303 & 1.17159 & 1.17161 & $7.6 \cdot 10^{-7}$ & $4.3 \cdot 10^{-8}$ & 465.324 & 465.262 \\
\hline & $\mathrm{FE}$ & 303 & & 1.17049 & & $4.8 \cdot 10^{-4}$ & & 476.651 & \\
\hline
\end{tabular}

In Figures 810 the normalized curves of the radial displacement, radial and tangential stresses can be seen by 5 different volume fractions $(N=0.16,0.3,1,3,6)$.

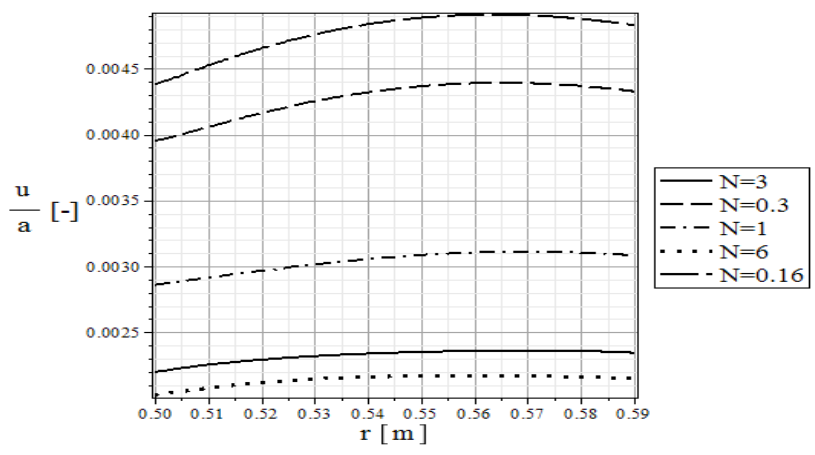

Figure 8. The normalized radial displacements by different volume fractions

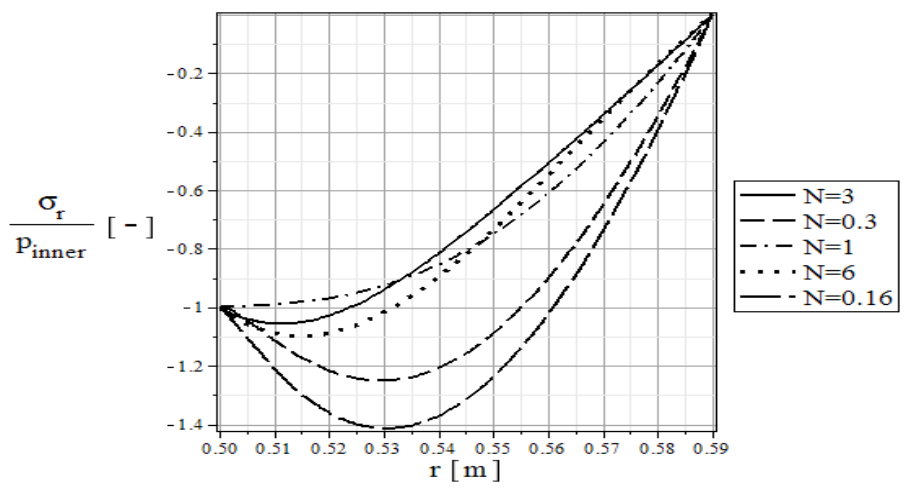

Figure 9. The normalized radial stresses by different volume fractions 


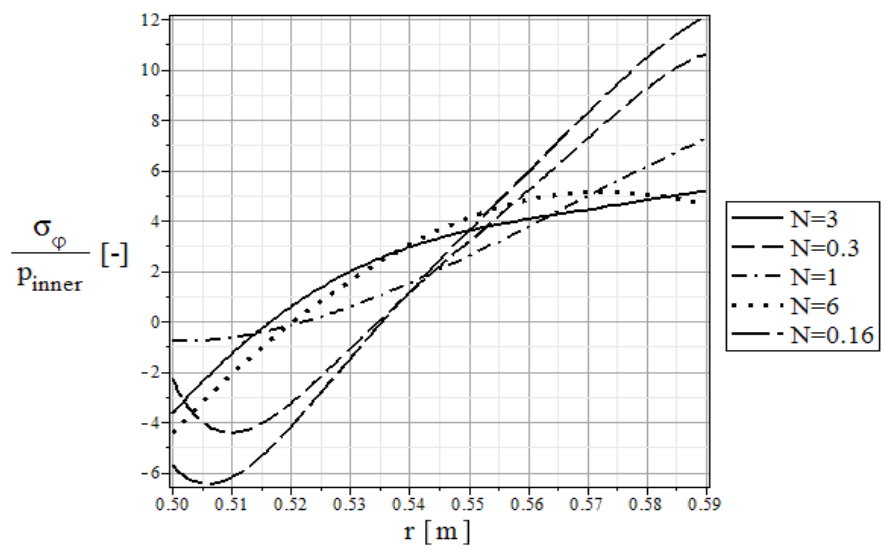

Figure 10. The normalized tangential stresses by different volume fractions.

\section{Conclusions}

The main objective of this paper has been to presents a numerical solution for the determination of the displacement field and normal stresses in functionally graded hollow spheres subjected to spherically symmetric thermal and mechanical loads. The material properties of the FGM are arbitrary functions of the radial coordinate and the temperature field. In the developed method the radially graded spherical pressure vessel has been modeled as a multilayered spherical body, furthermore Fourier's law of heat conduction and the equations of thermal stresses with steady-state temperature field have been used. Analytical solutions have been derived to check the accuracy of the method and the results have been compared to finite element simulations. The developed method can be utilized during the designing of the material composition along the radial coordinate for FGM spherical pressure vessels or as benchmark solutions to verify the accuracy of other numerical methods.

\section{REFERENCES}

1. Boley, B. A. and Weiner, J. H.: Theory of Thermal Stresses. John Wiley \& Sons Inc., New York, 1960.

2. Lekhnitskit, S. G.: Theory of Elasticity of an Anisotropic Body. Mir Publishers, Moscow, 1981.

3. Lomakin, V. A.: Theory of Nonhomogeneous Elastic Bodies. MGU, Moscow, 1976.

4. Lutz, M. P. and Zimmerman, R.W.: Thermal stresses and effective thermal expansion coefficient of a functionally graded sphere. Journal of Thermal Stresses, 19, (1996), 39-54.

5. Tutuncu, N. and Ozturk, M.: Exact solutions for stresses in functionally graded pressure vessels. Composites, Part B, 32, (2001), 683-686. 
6. Lengyel, Á. J. and ECSEDI, I.: Static and dynamic analyses of composite beams with interlayer slip. Journal of Computational and Applied Mechanics, 10(1), (2015), 25-40.

7. Kiss, L. P. and SzeidL, Gy: Vibrations of pinned-fixed heterogeneous circular beams pre-loaded by a vertical force at the crown point. Journal of Sounds and Vibrations, 393C,(2017), 92-113.

8. KIss, L. P.: Green's functions for nonhomogeneous curved beams with application to vibration problems. Journal of Computational and Applied Mechanics, 12(1), (2017), $21-43$.

9. Lengyel, Á. J. and ECSEDI, I.: An analytical solution for two-layered composite beams with imperfect shear interaction. International Review of Mechanical Engineering, 10(7), (2016), 508-517.

10. ОватA, Y. and Noda, N.: Steady thermal stress in a hollow circular cylinder and a hollow sphere of a functionally gradient materials. Journal of Thermal Stresses, 7, (1994), 471-487.

11. Kim, K.S. and NodA, N.: Green's function approach to unsteady thermal stresses in an infinite hollow cylinder of functionally graded material. Acta Mechanica, 156, (2002), $61-145$.

12. GönCZI, D. and ECSEDI, I.: Thermoelastic analysis of functionally graded hollow circular disk. Archieve of Mechanical Engineering, 62(1), (2015), 5-18.

13. Eslami, M. R., Babaei, M. H. and Poultangari, R.: Thermal and mechanical stresses in a FG thick sphere. International Journal of Pressure Vessels and Piping, 82, (2005), 522-527.

14. Nayak, P., Mondal, S. C. and Nandi A.: Stress, strain and displacement of a functionally graded thick spherical vessel. International Journal of Engineering Science and Technology, 3(4), (2011), 2660-2671.

15. Bayat, Y., Ghannad, M. and Torabi, H.: Analytical and numerical analysis for the FGM thick sphere under combined pressure and temperature loading. Archive of Applied Mechanics, 10, (2011), 229-242.

16. Nowinski, I.L.: Theory of Thermoelasticity with Applications. Sythoff and Noordhoff, Alpen aan den Rijn, 1978.

17. Shen, H. S.: Functionally Graded Materials: Nonlinear Analysis of Plates and Shells. CRC Press, London, 2009.

18. Reddy, J. N. and Chin, C. D.: Thermomechanical analysis of functionally graded cylinders and plates. Journal of Thermal Stresses, 21, (1998), 593-626.

19. Mori, T. and TANAKA, K.: Average stress in matrix and average elastic energy of materials with misfitting inclusions, Acta Metallurgica, 21, (1973), 568-582.

20. Touloukian, Y. S., Gerritsen, J.K. and Moore, N. Y.: Thermophysical Properties Research Literature Retrieval Guide. Springer, 1967. 